

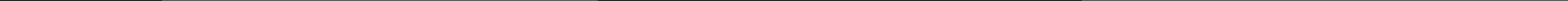




\section{El trabajo en el sector rural: dilemas y lógicas para un escenario de posacuerdo ${ }^{1}$}

Por Eugenio Castaño ${ }^{2}$

\section{Introducción}

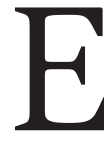

n medio de las favorables noticias sobre el cese al fuego bilateral entre el Gobierno y las FARC-EP es esencial construir nuevos espacios de concertación e inclusión social y laboral que tomen en consideración las dificultades padecidas por un sector históricamente relegado, como lo es el sector rural colombiano. Si bien para la Escuela Nacional Sindical es fundamental examinar las peculiaridades del trabajo rural, también es cierto que la naturaleza compleja de dicho trabajo obliga abordar dichas especificidades laborales desde un punto de vista social y económico mucho más amplio, con el fin de comprender las dinámicas y los retos que aquel sector entraña para el futuro del país en el mediano y largo plazo. En ese caso, el primer punto de los acuerdos de la Habana titulado Hacia un nuevo campo colombiano: Reforma rural integral, sumado a los dos últimos Planes Nacionales de Desarrollo, son, en teoría, las hojas de ruta sobre las cuales se ha desarrollado el modelo de "progreso" y de trabajo en el sector rural, de cara a los escenarios del posacuerdo.

$1 \quad$ Artículo recibido en agosto de 2106.

Artículo aprobado en noviembre de 2016.

2 Licenciado en Ciencias Sociales de la Universidad de Caldas, Manizales. Magister en Historia de la Universidad Nacional de Colombia, Sede Medellín y estudiante de Doctorado en Historia de la Universidad Nacional, Sede Medellín. 
A la luz de dichas apreciaciones y contexto, este artículo pretende llevar a cabo un análisis comparativo entre el primer punto de la Habana, referido al tema agrario, y los dos últimos PND del presente gobierno de Juan Manuel Santos. El artículo vuelca la atención sobre las incongruencias existentes entre lo formulado en el acuerdo y lo planteado en los PND, además de trazar unas recomendaciones incipientes para diseñar una política pública de trabajo decente e incluyente en el sector rural colombiano.

\section{Perspectivas para fin del conflicto armado}

Según lo planteado por Reyes (2010), dos razones llevaron al Gobierno a la convicción de sentarse en una mesa de negociación con las FARC-EP y llegar a acuerdos en materia rural. En primer lugar, una reforma rural integral se constituía como una vía rápida hacia la seguridad alimentaria, la eficiencia económica y la igualdad social. En segundo lugar, el escenario de diálogo también era una oportunidad política excepcional para realizar la tarea pendiente del desarrollo, con la cual se garantizarían los derechos de empresarios y campesinos. La negociación de aquel primer punto se demoró diez ciclos, de once días cada uno, entre el 28 de octubre de 2012 hasta el 26 de mayo de 2013. Fueron muchas las dificultades presentadas en esta primera etapa, entre las cuales había dos aspectos fundamentales. Por un lado, las FARC-EP se resistían a reconocer en el Gobierno nacional un agente capaz de agenciar una reforma rural profunda que cerrara la brecha entre el mundo rural y el urbano. Por otro lado, el Gobierno tampoco reconocía en aquel grupo insurgente un actor legítimo que reivindicara las demandas populares en provecho del sector rural.

A ello se sumó una discusión profunda en torno a dos miradas diferentes. Para el grupo guerrillero, tal como lo señala Reyes Posada (2016), la expansión del latifundio y la exclusión violenta del campesinado, la amenaza de los biocombustibles y los hidrocarburos, la adquisición de 
tierras por parte de las multinacionales, entre otros asuntos, se constituyeron en factores claves que alimentaron el conflicto armado. En vista de lo anterior, el grupo insurgente de más trayectoria en el país defendía la necesidad de diseñar un tipo de economía agraria familiar, además de brindarle un impulso definitivo a las zonas de reserva campesina. De otro lado, para el Gobierno, el campo era vulnerable al conflicto armado y al narcotráfico; de modo que, según la visión oficial, el atraso rural y la violencia iban inextricablemente unidas. Así pues, era necesario centrar los esfuerzos en el bienestar de las personas y la integración de territorios abandonados con un enfoque de desarrollo completo, capaz de generar fuentes de ingreso decente.

\section{Acuerdos en materia rural, dhacia un nuevo campo colombiano?}

En el mes de junio del 2014 se publicaron algunos puntos acordados en la Habana en los que se hacía mención de cuatro aspectos fundamentales para saldar la deuda histórica que se tenía con el sector rural en el país. El primero de estos puntos, que llamaremos punto A, se desglosó en una serie de subtemas. Estos señalaban primordialmente un acuerdo sobre el establecimiento de un fondo de tierras de distribución gratuita para los campesinos sin tierra o con tierra insuficiente; ello implicaba acceso integral a la tierra, acompañada de riego, crédito, asistencia técnica y apoyo para la comercialización.

Sin embargo, existían dificultades enormes, ya que el otorgamiento de las tierras provendría fundamentalmente de los baldíos debido a aspectos como los costos en el financiamiento para inventariar las tierras en Colombia. Este punto está fuertemente ligado a la dificultad de formalizar y titular las hectáreas ocupadas por los colonos en zonas de frontera, porque, como señala Londoño (2013), la recuperación de baldíos ilegalmente apropiados era un proceso bastante dilatado, tanto en términos administrativos como legales. 
Además, existía un claro interrogante frente al hecho de plantearse de dónde provendrían aquellas tierras ocupadas. En ese caso, también estaban los bienes de extinción de dominio, los cuales habían demostrado durante largos años ser una fuente de retrasos y de obstáculos jurídicos a la hora de volver a adjudicar aquellos predios extinguidos o incautados a los campesinos. Algunos sectores sociales revelaron que un porcentaje de dichos predios, que harían parte de aquel banco de tierras, eran utilizados para otros fines comerciales, como carbón, o bien eran dedicados a los monocultivos de la palma. Frente a esta situación se hizo compleja la implementación de una política de restitución de tierras en favor de los campesinos desalojados de sus predios por los grupos armados, a pesar de que la cantidad de dichos terrenos abandonados o despojados ha sido muy elevada.

El fondo que buscaba restituir tierras al campesinado debería disponer de tierras suficientes para, solo en principio, proveer a doscientas cincuenta mil familias aproximadamente. Ello sin contar con las familias que solo disponían de media unidad agrícola familiar o fueron despojados de sus tierras por cuenta del conflicto armado. También estaban quienes habían abandonado un total de 270.680 predios, es decir, 6.556 .968 hectáreas aproximadamente, sumadas a las 1.500 .000 hectáreas declaradas por personas desplazadas antes del 2004; tierras que no estaban procesadas. En síntesis, según el ministro de Agricultura, Aurelio Iragorri, el fondo no solo incluiría terrenos baldíos, sino también quinientas mil hectáreas confiscadas al narcotráfico en las mejores regiones. Adicionalmente, existía un problema logístico sobre cómo brindar tierras para los campesinos minifundistas, ubicados en las zonas centrales del territorio nacional, donde no solían existir baldíos del Estado para distribuir. Este asunto certificaba el peso que acarreaba la concentración de la tierra en el país, pues el $41 \%$ de esta estaba en manos de los grandes propietarios, el 40\% en manos de medianos propietarios y el 19\% restante estaba en poder de pequeños propietarios, minifundistas y microfundistas ${ }^{3}$.

3 Foro departamental de seguimiento a los acuerdos de la Habana, 2016. 
El segundo subtema del punto A se enfocó en el ofrecimiento de un subsidio integral y crédito especial para compra de tierras. En ese caso, se acordó impulsar un plan masivo de formalización de la pequeña y mediana propiedad, así como la creación de una jurisdicción agraria con el fin de asegurar la protección judicial a los derechos de dichas propiedades. Con ello, como dijo en 2014 el Alto Comisionado para la Paz, se buscaba impedir nuevos procesos de desplazamiento. En definitiva, este acuerdo intentó optimizar el uso de la tierra para mejorar las fuentes de ingreso, poniendo en marcha programas de reconversión, formación y actualización del catastro. De acuerdo con lo planteado por Lucía Londoño (2013), aquel último aspecto, es decir la regulación de tierras vía catastro, entrañaba un significado realmente importante porque las irregularidades de esta índole generaban inequidad en los avalúos y, por consiguiente, en el pago del impuesto predial. Según la investigadora, el avalúo catastral de una hectárea, ubicada en una gran propiedad, era siete veces menor que el avalúo en un microfundio, tres veces menor que el avalúo en una pequeña propiedad y 3,3 veces menor que el de una mediana propiedad. Por consiguiente, la actualización del catastro permitiría, en principio, saber realmente cómo estaba distribuida la tierra en Colombia; además de construir un nuevo escenario para mejorar la financiación de los municipios.

Según Guzmán (2014), el segundo punto del acuerdo, al que mencionaremos como punto $\mathrm{B}$, en materia rural se orientó al establecimiento de Programas de Desarrollo con Enfoque Territorial (PDET). El propósito era reconstruir las regiones más afectadas por el conflicto, aumentar el área sembrada, reducir la pobreza y ajustar la política rural conforme con los retos impuestos por las nuevas condiciones ambientales. En efecto, como dio cuenta El Nuevo Siglo en 2014, en departamentos como el Tolima, la presentación del Programa de Desarrollo Rural procuraba adelantar estrategias productivas en alianza con el Incoder y bajo el marco del Contrato Plan Sur del Tolima. De allí se desprendió la necesidad de diseñar planes de desarrollo con capacidad para transformar los territorios, garantizar derechos y facilitar escenarios de reconciliación. La impronta trazada por 
este punto del acuerdo se centró en la construcción participativa de planes de acción para la transformación regional y el mejoramiento de las condiciones sociales y de ingreso campesino. El tercer punto, es decir el C, hizo hincapié en los Planes Nacionales de Desarrollo, pero esta vez con énfasis marcado en la reducción de la pobreza y de la pobreza extrema. Dichos planes debían proveer bienes y servicios públicos en infraestructura, desarrollo social y estímulos a la productividad de la agricultura familiar y la formalización laboral. Finalmente, en cuarto lugar, el punto $\mathrm{D}$ de los acuerdos se dirigió hacia el establecimiento de un sistema especial de seguridad alimentaria y nutricional, con el fin de robustecer los mercados regionales y locales.

Ahora bien, en relación con lo anterior, el nuevo acuerdo en materia rural introdujo un Sistema General de Información Catastral, integral y multipropósito, que en un plazo de siete años concretara la formación y actualización del catastro, ejecutado en el marco de la autonomía regional. También se mencionó en el nuevo acuerdo el otorgamiento de subsidios para que los municipios más débiles y los predios más pequeños pudieran contar con catastros actualizados, en espera de que se implementara en los municipios más afectados por la violencia. De igual forma, se redefinieron las reservas forestales que tuvieran vocación agrícola, sin concederles poderes especiales a las comunidades.

Hubo otros dos aspectos llamativos del nuevo acuerdo en materia rural. El primero de ellos fue el reconocimiento explícito de la propiedad privada, lo que conllevó, como seña la Kalmanovitz (2016), la articulación entre la gran economía agrícola y la pequeña economía campesina, restándosele así importancia a las Zonas de Reserva Campesina. En segundo lugar, se introdujo el principio de Desarrollo Integral del campo, entendido este como el balance entre las diferentes formas de producción existentes: agricultura familiar, agroindustria, turismo, agricultura comercial de escala ${ }^{4}$.

$4 \quad$ Foro departamental de seguimiento a los acuerdos de la Habana, 2016. 
Finalmente, en lo concerniente al tema de formalización laboral, el acuerdo propuso el fortalecimiento del sistema de protección social para los trabajadores rurales a través de un plan progresivo. Con dicho plan se pretende, en teoría, dignificar las condiciones de trabajo rurales mediante la aplicación plena, con la inspección del trabajo, de la normatividad existente sobre relaciones contractuales, regulación sobre jornada laboral, remuneración y subordinación. También se mencionan una serie de criterios para su puesta en marcha: campañas para la erradicación del trabajo infantil, garantía de protección social, estimulo de procesos organizativos, vinculación laboral de personas en situación de discapacidad, vinculación de mujeres en áreas productivas no tradicionales, capacitación a trabajadores y trabajadoras en materia de obligaciones y derechos laborales, fortalecimiento de las inspecciones laborales, programas social y ambientalmente sostenibles en las zonas rurales, protección del riesgo económico de la vejez hacia la población rural en extrema pobreza, esquemas de protección al embarazo, parto, lactancia y atención en salud para el recién nacido. También se propone, por un lado, el fomento de la asociatividad, es decir de los encadenamientos y alianzas productivas entre pequeños, medianos y grandes productores, así como con procesadores, comercializadores y exportadores. Por otro lado, se propone la puesta en marcha de un sistema para la garantía progresiva de un sistema de alimentación. Estos dos últimos puntos están enfocados en garantizar una producción a escala, competitiva, insertada en cadena de valor agregado y que contribuya a mejorar las condiciones de vida de los habitantes rurales ${ }^{5}$.

\section{Retos e interrogantes frente a la implementación de los acuerdos}

Para Reyes (2010), con este acuerdo se pretendió que el nuevo desarrollo rural con enfoque territorial tuviese implicaciones concretas en el

Acuerdo final para la terminación del conflicto y la construcción de una paz estable y duradera, 2016. 
ordenamiento territorial. En ese caso, serían las propias condiciones de los territorios las que determinarían la exigencia de coordinación de las agencias públicas alrededor de los programas de desarrollo, ejecutados por los Comités Locales. Para ello, a su vez, se definieron áreas priorizadas, una vez se llegase a un acuerdo definitivo con las FARC-EP. Con ello se buscaba implementar una estrategia orientada a focalizar los programas en los territorios, a través de la coordinación de esfuerzos y competencias en el nivel central, regional y local. Incluso, también se afirmaba que el enfoque territorial debía conducir al diseño de un catastro que permitiese, realmente, hacer un inventario de las tierras existentes en Colombia. El interés es poder dar cuenta de las tierras pertenecientes al sector privado, las pertenecientes a los grupos étnicos, indígenas y afrocolombianos, además de aquellas destinadas a protección y recursos naturales.

En ese sentido, existen algunas experiencias adelantadas, como la llevada a cabo en el departamento de Antioquia, en donde se han desarrollado procesos de fortalecimiento territorial, comunitario y de incidencia institucional en algunas subregiones. Detrás de estas propuestas y planes está la intención de realizar un mapeo capaz de promover estrategias de empoderamientos en las comunidades campesinas y de facilitar procesos de formalización de las tierras. A partir de ahí, se busca identificar a los actores en el territorio, impulsar procesos sobre apropiación de conocimientos cartográficos y análisis de catastros. Adicionalmente hay una pretensión de democratizar la información, conocer de primera mano la ubicación de los baldíos, verificar coordenadas y establecer líneas de acción desde lo local. Así, cuando la Agencia Nacional de Tierras haga presencia en la zona, la idea es tener mayor claridad sobre la forma de intervenir y diseñar políticas en provecho de los pobladores locales y sus formas de concebir y optimizar su trabajo ${ }^{6}$.

6 Foro departamental de seguimiento a los acuerdos de la Habana, 2016. 
Por otro lado, en La Habana también se planteó la creación de Consejos de Seguridad Alimentaria y Nutricional en todos los niveles territoriales como un tema a tratar con urgencia. Además, por supuesto, se abordó el tema crucial del narcotráfico que, aunque correspondía a otro de los puntos del acuerdo con el grupo insurgente, tenía una enorme implicación en lo referido al tema de la producción y los ingresos. Este último era un punto bastante problemático para algunos sectores campesinos, toda vez que un porcentaje amplio de ellos sembraba hoja de coca y amapola para su supervivencia, al tiempo que no existían claridades respecto a lo que se concebía por seguridad alimentaria. Por tanto, la sustitución de lo que oficialmente se ha denominado cultivos ilícitos debía llevarse a cabo de manera concertada con las comunidades, aunado a un proyecto económico y laboral lucrativo y sostenible en el tiempo. En ese sentido, se denunciaba el alto costo de fabricación de productos alimenticios, de los insumos agropecuarios y del transporte a los sitios de comercialización, lo cual contrastaba con la alta rentabilidad de aquellos cultivos tipificados como ilegales.

A ello se le añade el temor de que con la puesta en marcha de las políticas de sustitución por cultivos “legales”, el acompañamiento a los campesinos, por parte del Estado colombiano y a través de subsidios paliativos, fuese temporal. Alrededor de ello se planteaban los siguientes interrogantes: ¿A largo plazo, qué pasaría con estos campesinos después de firmados los acuerdos?, ¿de nuevo quedarían a merced de los vaivenes del mercado?, ¿qué sucedería después cuando la coyuntura de la paz dejara de ser un asunto clave dentro de las agendas de gobierno?, ¿qué ocurriría con aquellos campesinos que durante el proceso de transición hubiesen decidido sustituir sus cultivos ilícitos?, ¿el Estado colombiano estaría dispuesto a realizar un acompañamiento a largo plazo, a través de políticas estructura les, que beneficiaran a los campesinos que decidieran modificar sus fuentes de ingreso? ${ }^{7}$

Foro departamental de seguimiento a los acuerdos de la Habana, 2016. 
Los anteriores cuestionamientos planteaban un reto enorme, no solo a la política de sustitución de cultivos ilícitos, sino al corazón mismo de la reforma rural. Existía una discusión de fondo sobre la situación económica del campesinado colombiano en relación con las oportunidades de trabajo y con las posibilidades reales de eliminar los factores de pobreza. En suma, se planteaba que la devolución de la tierra, por sí sola, no sería suficiente para mejorar las condiciones de vida de este sector poblacional. Dicho en otros términos, devolver la tierra sin mayores atenciones y precauciones podría facilitar el retorno a un estado de pobreza anterior al despojo: "A un campesino le retornan su tierra y como no tiene los recursos ni el acompañamiento para hacerlas productivas, pues terminan vendiéndola, porque que más va a hacer” (Entrevista a líder campesino de Dignidad Agropecuaria, 2016). A ello se le adicionan los testimonios de otros campesinos, para quienes el despojo había provocado la incorporación de muchos de los pobladores rurales a los grupos armados, pues se veía como una opción de “trabajo” frente a la ausencia de otras posibilidades de empleabilidad dentro de las "vías legales".

Otros sectores vinculados con el Centro Democrático (2014) tejieron todo un manto de dudas sobre los alcances de los acuerdos, al calificarlos como una forma de proponer la colectivización socialista de la tierra, poniendo en riesgo, según ellos, la propiedad privada. Pero para el Gobierno, aquellos acuerdos no conducían necesariamente a la colectivización de la tierra. Antes bien, lo que se formulaba era la necesidad de recuperar tierras adquiridas por medios ilícitos, así como los baldíos obtenidos por medio de mecanismos fraudulentos.

Frente a este último aspecto es importante hacer una lectura más detenida.

Si bien el argumento del Gobierno se apoyó en la necesidad de generar una mayor apertura de la tierra como fuente de ingreso para los más pobres, en ningún momento, dicho argumento, se opuso al principio de la 
propiedad privada como bien supremo. Con ello, finalmente, se reafirmó la legitimidad política de la propiedad, evitando centrar la discusión en torno a la inminencia de una supuesta reforma agraria profunda. Incluso la tesis del Gobierno se valió del principio de la formalización de la propiedad como una herramienta destinada a brindar mayores garantías jurídicas. Se trataba de brindar facilidades a los procesos de comercialización de las tierras, además de garantizar inversiones sostenibles en el campo. Ello no necesariamente entrañaba una protección directa al pequeño campesino para resistir a las arremetidas de los grandes empresarios de la tierra.

Si bien es cierto que la informalidad en los títulos de propiedad facilitó el despojo a través del ejercicio de la violencia, también es cierto que la formalización de la propiedad rural estaba en capacidad de allanar el camino para su comercialización en el mercado, y no necesariamente para la redistribución equitativa de las mismas entre los campesinos más pobres.

Para ilustrar lo anterior, en un evento denominado "Expo Agrofuturo", llevado a cabo en la ciudad de Medellín a finales del 2014, el Superintendente de Notariado y Registro, Jorge Enrique Vélez, puso de presente la importancia de brindar seguridad jurídica a las inversiones en tierras. Dos años atrás, como anota Rojas (2012), el entonces Ministro de Agricultura, Juan Camilo Restrepo, ya había planteado la necesidad de reducir la inseguridad jurídica para regular, de una manera más adecuada y eficiente, la inversión extranjera. Lo interesante es constatar que dicha apuesta parecía estar articulada con la necesidad de proveer tierras, puestas a merced de los juegos de un mercado que, a su vez, debía estar amparado por unos mecanismos jurídicos bastante sólidos. Por consiguiente, como dice Estrada (2014), la idea de construir una "nueva ruralidad" no se alejaba radicalmente de las clásicas ideas articuladas con la búsqueda de rentabilidad, valor agregado y productividad con visión empresarial. 
En suma, si con lo planteado en los acuerdos el Centro Democrático señalaba el riesgo de atentar contra la propiedad rural privada como principio constitucional, el Gobierno declaraba que su accionar se ajustaba a lo estrictamente demarcado por la misma carta constitucional. Igual sucedía con otro tipo de señalamientos en torno a las presuntas concesiones otorgadas a las FARC-EP.

Por un lado, se le criticaba al gobierno su claudicación a través del favorecimiento de las Zonas de Reserva Campesina; pero, por otro lado, el Gobierno respondía que dicha figura era bastante tradicional dentro de los propios lineamientos normativos nacionales, los cuales se remontan hasta1994. En ese sentido, es curioso que, al revisar los acuerdos de La Habana, se acogiera la figura de las zonas de reserva campesina. Sin embargo, para la Asociación Nacional de Reserva Campesina, Anzorc, estas intenciones parecían enlodarse frente a los paquetes legislativos preparados para, según afirmaban, entregar los baldíos al gran capital. El temor manifestado por dicha Asociación radicaba en la presunta aniquilación de la Unidad Agrícola Familiar y de los mecanismos de consulta a las comunidades campesinas, indígenas y afros. A esta situación se le sumaba la preocupación por las constantes estigmatizaciones padecidas por parte de entidades como el Ministerio de Defensa, algunos Senadores y Representantes a la Cámara, además de las históricas amenazas provenientes de los grupos armados.

Si bien los planes del Gobierno quisieron imponer su sello a través de la reivindicación de principios como la equidad, el combate contra la pobreza rural y una mayor democratización de la tierra como fuentes de ingresos dignos y decentes, también es cierto que jamás se puso en tela de juicio el modelo de desarrollo en su conjunto. Si el Centro Democrático enfiló baterías contra el Gobierno con el objetivo de perturbar la buena marcha de los mecanismos políticos y económicos del país, bajo los cauces de un supuesto principio socialista, dicho principio puso de presente su carácter moderado en los alcances como una manera de evitar la animadversión de un amplio sector de la élite regional y 
nacional. Ello se veía reflejado en situaciones como la presentada por un conjunto de parlamentarios de la Alianza Verde, quienes indicaban que el Gobierno nacional estaba dando rienda suelta a las "licencias exprés en favor de las multinacionales, sin poner en consideración la protección a las fuentes hídricas y los páramos” (El Espectador, 2015).

Existen ciertos temores frente a las irregularidades en la adquisición de predios agrícolas por parte de los grandes grupos empresariales, lo cual contribuía a acentuar el debate sobre el modelo de desarrollo agrícola en el país y, por ende, el tipo de empleo rural que se deseaba impulsar. De allí que emergieran, por un lado, posturas en donde se promoviera una reforma agraria integral y la economía campesina, además del impulso a una política de restitución de tierras; y, por el otro, una perspectiva oficial, ajustada a las dinámicas de los tratados de libre comercio y a los proyectos agroindustriales. No obstante, como deja ver De la Torre (2012), la paradoja sembrada entre las intenciones por restituir las tierras en beneficios de los pequeños campesinos y las necesidades de asentir a las exigencias de dichos tratados, ponía sobre el tapete la urgencia de crear mecanismos de compensación que ofrecieran opciones de supervivencia y de trabajo digno e incluyente para los habitantes del campo; lo cual debía darse, además, con el fortalecimiento de las zonas de reserva campesina. A la luz de lo anterior, la decisión de subsidiar la producción de ciertos proyectos agroindustriales como los biocombustibles, sumado a la vasta extensión de tierra subutilizada y dedicada a la ganadería extensiva, no solo desembocó en la reducción de la oferta alimentaria, sino que también, como anota Hommes (2011), desencadenó un alza en sus precios y del desempleo agrícola en los últimos años.

La Superintendencia de Notariado y Registro advirtió sobre las "artimañas” utilizadas para robarle al Estado los lotes baldíos de los que disponía, en detrimento de los intereses de los campesinos más pobres. En departamentos como el Vichada, existían dos interrogantes en cuanto a la naturaleza de los procesos de poblamiento. Primero, aquella duda estribaba en conocer si, efectivamente, esta zona era poblada por 
campesinos pobres en busca de un mejor proyecto de vida; o si, por el contrario, el apremio colonizador ponía al descubierto un nuevo proceso de concentración de la tierra y de precarización laboral en perjuicio de los campesinos más pobres. En efecto, para el Superintendente de Notariado y Registro, Jorge Enrique Vélez, muchos propietarios de lotes en esta zona del país eran citadinos que nunca habían vivido en la zona. De acuerdo con él, "pese a hacerse con el negocio de sus vidas, no les importan los proyectos productivos”.

Según Delgado (2013), en estos sucesos había una ausencia de controles por parte del Estado, lo que generaba vacíos en los procesos de restitución y apropiación de predios, así como de seguimiento de acuerdo al uso que se le estaba dando a las tierras. Las irregularidades más comunes eran aquellas en las cuales se hacía una sola escritura para varias UAF (Unidad Agrícola Familiar). Es decir: varias empresas de un mismo grupo económico adquirían una UAF y las unificaban; o bien se recurría al despojo y a la creación de sociedades, cada una con un NIT diferente, para poder acceder al mismo tiempo a varias UAF. En segundo lugar, algunos expertos opinaban que no era precisamente en Vichada o Meta donde los baldíos debían ser usados en beneficio de los campesinos. Todo ello, como dice Botero (2014), teniendo en cuenta que allí los suelos eran extremadamente pobres en nutrientes y además costaba una fortuna habilitarlos para la producción.

\section{Principios y contradicciones de los PND 2010-2014 y 2014-2018}

Este panorama examinado hasta ahora permite lanzar un interrogante sobre los alcances reales del primer punto de los acuerdos de La Habana, además de las contradicciones existentes entre lo planteado allí, con lo propuesto en los Planes Nacionales de Desarrollo. En principio, si por un lado la reivindicación de programas como la "seguridad democrática”, "confianza inversionista” y "cohesión social” han contribuido 
a profundizar el fenómeno de las titulaciones mineras y la incorporación de las tierras a usos presuntamente más productivos; por otro lado, la cosmovisión de un amplio sector del campesinado nacional se orienta hacia una profunda reafirmación de la vida en contra de la precarización laboral, la concentración de la tierra, la exclusión social y la devastación de los ecosistemas.

Algunos sectores comenzaron a albergar serias dudas sobre las contradicciones entre lo establecidos en los PND y lo planteado en los acuerdos de La Habana. Organizaciones como el Cinep, Codhes, Mesa de Incidencia Política de las Mujeres Rurales Colombianas, Comisión Colombiana de Juristas, Dignidad Agropecuaria y Cumbre Agraria expusieron sus reservas sobre el tema de baldíos, ordenamiento territorial y paz. Para estas instituciones y organizaciones el tema de los baldíos en los PND no reconocía a los pueblos étnicos y campesinos que los habitaban y usufructuaban, lo cual negaba su función social y abría la posibilidad para que fuesen entregados a empresas privadas. Ello daría rienda suelta a la posibilidad de fomentar inversiones mineras y agroindustriales en las tierras baldías, como estrategia para mejorar las escalas productivas.

Para estas voces, revestidas de carácter crítico, el concepto de "eficiencia” cobraba un sentido particular al articularse al peso de las estructuras empresariales, lo cual ponía en un lugar bastante desventajoso al campesinado pobre. El mejoramiento del entorno "competitivo" para los negocios se tradujo en una mayor oferta de recursos naturales para la agroindustria, oferta que, como bien muestra Zambrano (2015), se vio reflejada en la existencia, solo para el mes de mayo del 2015, de 120 multinacionales interesadas en hacer negocios y de 220.000 hectáreas habilitadas en el departamento de Cundinamarca. Para ilustrar lo anterior basta con mencionar lo sucedido en el 2013 cuando el entonces embajador de Colombia ante Estados Unidos renunció a su cargo, como respuesta frente al gran escándalo desatado por la adjudicación 
de tierras en el departamento de Vichada, en favor de la empresa Cargill Riopaila; tierras que en principio estaban destinadas a los campesinos.

En ese sentido, los acuerdos de La Habana sobre el desarrollo agrario integral y las bases de los dos últimos PND parecen ser la puerta de entrada respecto al enfoque asumido para el desarrollo del sector agropecuario, de cara a los escenarios de un posible posacuerdo. En cualquier caso, queda un interrogante abierto: ¿cómo conciliar estas realidades vinculadas con el complejo mundo del trabajo rural, además de los objetivos propuestos en aquellos acuerdos, con lo promulgado en los dos últimos Planes Nacionales de Desarrollo?

En principio, en el PND 2010-2014 se mencionaron las estrategias, rutas y esquemas operativos para conformar Áreas de Desarrollo Territorial, además de crear ejes o nodos articuladores capaces de conectar los polos de desarrollo para la creación de entornos territoriales competitivos y generadores de empleo. Por ejemplo, se planificó la construcción de los ejes Bogotá-Villavicencio-Arauca y Bogotá-Villavicencio-San José del Guaviare. Se aspiraba a que dichos ejes tuviesen funciones urbanas, pero que también pudiesen servir de apoyo para el fomento del empleo y el desarrollo agroindustrial, pecuario y de rutas eco turísticas en la región del país abarcada por dichas rutas. De allí la necesidad de establecer unos lineamientos estratégicos enfocados en el acceso a nuevos mercados globales, por medio de la optimización de las competencias laborales y la creación de nuevos empleos con el apoyo de instituciones formadoras como el SENA ${ }^{8}$. Según Correa (2011), a la luz de lo anterior se precisó que para el sector agrícola el Ministerio de Agricultura y Desarrollo Rural definiría los mecanismos para la ejecución de las agendas de innovación por cadenas productivas; además de hacer más rentable una serie de territorios históricamente desprovistos de inversión, como la Orinoquía. Más allá de este tipo de planteamientos, en el Plan de Desarrollo 2010-2014 no se dieron mayores análisis acerca del diseño

8 Departamento Nacional de Planeación, 2010. 
integral de nuevos empleos dignos, decentes e incluyentes, y con un enfoque diferencial y territorial. Tampoco se mencionó la necesidad de promover formas organizativas en el sector rural, como una manera de construir espacios de consenso y deliberación sobre temas atinentes al mejoramiento de las condiciones del trabajo y los ingresos rurales.

Adicionalmente, dentro del primer PND de la administración de Juan Manuel Santos existían algunos artículos que fomentaban un tipo de desarrollo municipal de carácter agrario y forestal; artículos que abrían la posibilidad de arrendar tierras baldías y acumular UAF adjudicadas a colonos. Con ello, según algunos sectores, se agudizaba el proceso de concentración de tierras, más aún cuando el gobierno mantenía en pie la idea de otorgar tierras en arriendo para los proyectos agroindustriales. Aquello reflejaba una clara intención por incorporar a Colombia dentro de los “mercados internacionales", mas no la intención de centrar esfuerzos en el componente social y las cosmovisiones que sobre la tierra poseían los campesinos. Lo anterior se ha visto reflejado en la negativa del Gobierno nacional a negociar el tema minero y de hidrocarburos en las discusiones y consultas sobre los PND.

Por otro lado, al analizar los alcances del PND 2014-2018, de nuevo se planteó la necesidad de transformar el campo; además, el tema de la paz parece ganar mayor protagonismo en comparación con el PND anterior. Por tal razón, se expresó la necesidad de llevar a cabo la transformación integral del sector rural colombiano, a través de la puesta en marcha de varios puntos relacionados con la educación rural, el cierre de las brechas en los ingresos urbano-rurales, la disminución de la pobreza rural, fomento de estándares elevados de competitividad, además de otros aspectos como formalización de tierras y mejoramiento de capacidades productivas. Sin embargo, y de manera semejante a lo vislumbrado en el anterior PND, no se advierte de manera minuciosa la forma de implementar políticas diferenciales de ingresos y empleos incluyentes y dignos, más allá de la mención a los proyectos agro-empresariales, erigidos en los motores del "desarrollo”. 
Las pocas indicaciones directas sobre la creación de nuevos empleos rurales se concentran, básicamente, en el sector minero energético. De igual forma, en el PND 2014-2018 se mencionó la necesidad de promover aquello que el gobierno denomina "el autoempleo rural” -sin hacer mayores precisiones sobre lo que ello significa-, el cual está destinado a beneficiar la población víctima del conflicto armado. De nuevo, en este punto, emergen los mismos interrogantes en referencia a los presuntos alcances de aquel "autoempleo rural", ya que no brindan mayores elementos de análisis respecto a la forma de articular aquello con las necesidades de incorporar unos mayores niveles de formalización, trabajo decente y apoyo a proyectos productivos en beneficio de la población rural más vulnerable ${ }^{8}$.

A lo anterior vale la pena agregar la ausencia de un debate abierto a la hora de replantear todo el corpus ideológico que contiene los PND. De acuerdo con lo afirmado por una lideresa sindical de Fensuagro, si bien parece plantearse la necesidad de construir una nueva institucionalidad rural dentro en un escenario para el posacuerdo, no deja de llamar la atención el hermetismo y la ausencia de espacios de concertación con las organizaciones campesinas y sindicales para replantear el tema de la explotación minera y petrolera, pilares del desarrollo económico del actual Gobierno. De igual forma, las reglas de la flexibilización laboral suelen someter a los trabajadores de las plantaciones agroindustriales a condiciones bastante precarias en términos de remuneración; muchos de ellos, incluso, carecen del derecho a seguridad social, además de estar sometidos a los mecanismos de la tercerización laboral. De allí que organizaciones como Fensuagro proclamen la necesidad de realizar consultas en los diferentes territorios rurales, como condición para la implementación de las políticas gubernamentales (Entrevista a lideresa de Fensuagro, 2015).

8 Departamento Nacional de Planeación, 2010. 


\section{Consideraciones finales}

Con base en lo examinado hasta ahora, se puede observar una enorme desconexión entre dos formas de concebir el desarrollo rural por parte del Gobierno y la forma en que lo aprecian los campesinos. Esta situación también se ve reflejada en las diferencias de enfoque apreciadas en el punto uno de La Habana y los PND, en la medida que el primero parece hacer hincapié en el componente social del debate sobre la estructura de las tierras y las economías campesinas, mientras que el segundo parece girar alrededor de un horizonte más articulado con las locomotoras del desarrollo agroempresarial. Por consiguiente, en los PND no se vislumbra, entre otras cosas, una política enfocada en atenuar las enormes disparidades de género engendradas en el campo colombiano. Así mismo, si bien parece existir un interés común en torno a la consolidación de un proceso de paz y de una política de desarrollo rural capaz de dar cuenta de los intereses de los campesinos más vulnerables -atendiendo a lo promovido en el acuerdo de tierras-, el Gobierno continúa amparándose en un estilo gerencial a la hora de abordar los asuntos del campo; un ejemplo de ello son los PND. En ese caso, esta esfera de acción parece ajustarse a las lógicas del mercado internacional y a unos parámetros supeditados al cumplimiento de estándares de competencia y eficiencia. Además, frente a las manifestaciones campesinas, las tramas de poder han mezclado la ilegalidad con las acciones de la fuerza pública y los grupos armados irregulares, como una especie de herramienta orientada a la contención de la protesta.

Igual acontece con las permanentes crisis desatadas por la precariedad institucional, la falta de coordinación entre los diferentes organismos gubernamentales y no gubernamentales, además de la corrupción, lo cual pone en crisis la concepción de ciudadano como sujeto de derechos. Si en el último PND se realizan algunas precisiones sobre los escenarios de paz en el sector rural, estas no son suficientes ya que está fuertemente marcada la ausencia de atención a la necesidad de fortalecer las organizaciones sindicales en el campo como forma de construir 
dichos escenarios. Es decir que hace falta también fortalecer el enfoque territorial. Adicionalmente, cuando se invoca el concepto de territorio en los Planes Nacionales de Desarrollo no es lo suficientemente clara la manera en la cual se articula dicho concepto con una profunda perspectiva histórico social, capaz de reconocer los múltiples significados culturales de los grupos que lo configuran, lo ocupan y lo usufructúan.

Si se toma en cuenta lo anterior, se torna indispensable que el Gobierno colombiano promueva estrategias inclusivas y diferenciadas para abordar la problemática del trabajo rural que atienda las diversas necesidades y demandas de los habitantes del campo. Para ello se requiere la apertura de espacios de reconocimiento e interlocución con las organizaciones sindicales rurales y el movimiento campesino, con el objetivo de diseñar los programas de gobierno que transformen las reales condiciones de trabajo en el sector rural colombiano. Lo anterior se señala tomando en cuenta aspectos básicos como: restitución de tierras, procesos de formalización laboral con enfoque de género, también, como anota con urgencia Salgado Araméndez (s/f), debe hacerse un reconocimiento de las economías y cosmovisiones e imaginarios campesinos. En ese mismo sentido de importancia es necesario fortalecer la inversión pública en infraestructura, el otorgamiento de créditos, subsidios y proyectos productivos en provecho del campesinado más precarizado.

En otras palabras, es necesario reformular la política agraria desde un enfoque territorial, de conformidad con los nuevos retos ajustados a los acuerdos de La Habana, que tenga en cuenta la necesidad de fortalecer la seguridad agroalimentaria y los ingresos de los pequeños y medianos productores, así como la necesidad de construir un estatuto del trabajo que pueda garantizar unos mayores niveles de inclusión en las relaciones laborales en el sector rural. En segundo lugar, es necesario impulsar políticas de formalización laboral con un enfoque de género y en beneficio de las y los asalariados sin tierra, que conduzca a una amplia cobertura de seguridad social, riesgos laborales y pen- 
sión. En tercer lugar se debe auspiciar políticas públicas que faciliten y salvaguarden los procesos sindicales y organizativos del campesinado colombiano durante los escenarios de posacuerdo, y por último, se deben diseñar estrategias encaminadas a mantener a la población joven en los diferentes territorios, además de minimizar los impactos de los procesos migratorios por falta de oportunidades laborales y formativas en las regiones.

Cada uno de estos aspectos requiere de una mirada transversal que dé cuenta de las dificultades que genera proponer una política de formalización laboral y trabajo decente, sin tomar en consideración las divergencias en los contextos de acción del campesinado nacional. Por ello se requiere, por un lado, el concurso directo y permanente del Estado colombiano para garantizar el éxito del proceso de formalización y de obtención de unos mínimos de trabajo decente, en provecho de los asalariados más precarizados y de los pequeños productores incapaces de solventar dichos gastos. Así como, por otro lado, resulta fundamental crear un equipo de trabajo con la participación activa del movimiento campesino y sindical, que evalúe los reales impactos económicos, sociales, culturales, ambientales y, por supuesto laborales, de la actual política rural nacional. Solo si se tienen en cuentan estas necesidades y exigencias urgentes de la situación agraria en el país, atendiéndolas de manera integral, el trabajo en el sector rural podrá dar cuenta de un escenario orgánico en términos de posacuerdo.

\section{Referencias bibliográficas}

Anónimo (18 de noviembre de 2016). Respuestas a las principales dudas sobre el nuevo acuerdo de paz. Recuperado de: http://www.altocomisionadoparalapaz. gov.co/Prensa/documentos-y-publicaciones/Documents/ Que_se_ha_acordado_en_La_Habana.pdf

Alto Comisionado para la Paz (junio de 2014). ¿Qué se ha acordado en la Habana? Recuperado de Asociación Nacional de Zonas de Reserva Campesina, Anzorc. El gobierno está dejando sin piso la posibilidad de implementar 
los acuerdos de La Habana en desarrollo agrario integral. Recuperado de http://prensarural.org/spip/spip.php?article16118

Botero Caicedo, M. (02 de noviembre de 2014). "Mitos y malentendidos agropecuarios”. En El Espectador.

Centro Democrático (22 de octubre de 2014). Las 68 capitulaciones de Santos en La Habana. Recuperado de http://www.centrodemocratico.com/ las68-capitulaciones-de-santos-en-la-habana-centro-democratico/

Correa, P. (01 de junio del 2011). “A la conquista del Orinoco”. En El Espectador.

De la Torre, C. (02 de octubre de 2012). “Dar tierra siembra paz”. En El Espectador.

Delgado, P. (02 de julio de 2013). "La reforma rural es una prioridad para el país”. En Vanguardia Liberal.

Departamento Nacional de Planeación. (2010). Bases del Plan Nacional de Desarrollo 2010-2014, Recuperado de http://www.cna.gov.co/1741/articles- 311056_PlanNacionalDesarrollo.pdf.

El Espectador (19 de febrero de 2015). Plan de Desarrollo contradice acuerdos en La Habana sobre la tierra, dicen ONG. Recuperado de http:// www. elespectador.com/noticias/politica/plan-de-desarrollo-contradice- acuerdos-habana-sobre-tie-articulo-544681

El Espectador (10 de julio de 2015). Piden al Gobierno no continuar otorgando "licencias exprés" para sector minero. Recuperado de http://www. elespectador.com/noticias/politica/piden-al-gobierno-no-continuarotorgando- licencias-expr-articulo-571722

El Nuevo Siglo (5 de septiembre del 2014). Fortalecen desarrollo rural en el Tolima.

El Tiempo (24 de julio de 2013). Lo que está detrás de la renuncia de Urrutia por escándalos de baldíos. Recuperado de http://www.eltiempo.com/ archivo/documento/CMS-12944905

Entrevista a líder campesino de dignidad agropecuaria (Mayo de 2016). Entrevista de Eugenio Castaño

Entrevista a lideresa de Fensuagro (Septiembre de 2015). Entrevista de Eugenio Castaño 
Estrada Ciro, J. (6 de septiembre del 2014). "Los empresarios no deben temer a invertir en tierras”. En La República.

Foro Departamental de Seguimiento a los Acuerdos de la Habana (Marzo de 2016). Medellín

Guzmán Hennessey, M. (05 de septiembre del 2014). "Astrolabio. Hacia buen puerto”. En El Tiempo.

Hommes, R. (06 de marzo de 2011). “La crisis de alimentos”. En El Colombiano.

Kalmanovitz, S. (27 de noviembre de 2016). "El nuevo acuerdo y una rectificación”. En El Espectador. Recuperado de: http://www.elespectador. com/ opinion/el-nuevo-acuerdo-y-una-rectificacion

Londoño, R. (03 de junio de 2013). "Acuerdo sobre el tema agrario: entre el presente y el futuro de la paz”. En Razón pública. Recuperado de http://www. razonpublica.com/index.php/conflicto-drogas-y-paztemas-30/6876-acuerdo-sobre-el-tema-agrario-entre-el-presente-y-elfuturo- de-la-paz.html

Presidencia de la República (22 de octubre [2014). Gobierno responde a las críticas infundadas a los acuerdos de La Habana.

Reyes Posada, A. (2016). La reforma rural para la paz. Bogotá: Debate.

Rojas, J. F. (20 de noviembre de 2012). "Minagricultura se la juega hoy para regular inversión extranjera en agro”. En El Colombiano.

Salgado Araméndez, C. (s/f). "Los campesinos imaginados” En Cuaderno Tierra y Justicia $\mathrm{N}^{\circ} 6$. Bogotá: Siglo del Hombre Editores.

Zambrano Triana, R. (13 de mayo del 2015). "Más de 120 multinacionales tras el agro nacional”. En La República. 